\title{
Un juego educativo online para médicos ayuda a que sus pacientes alcancen más rápidamente las metas de control de la presión arterial
}

\author{
An online education game among clinicians improves their patients' time to blood pressure control
}

Kerfoot BP, y col. Circulation. 2014;7(3):468-74

\section{Objetivos}

Evaluar la eficacia de un juego educativo online para médicos de atención primaria (MAP) como herramienta para disminuir el tiempo en alcanzar las metas de tensión arterial (TA) de sus pacientes hipertensos.

\section{Diseño}

Ensayo clínico aleatorizado, realizado en ocho hospitales de Estados Unidos, con una duración de 52 semanas (entre marzo del 2010 y abril del 2011). Los participantes fueron 111 MAP. Se realizó estratificación por hospital y aleatorización en bloque en dos cohortes: intervención (55) y control (56). Se realizó una evaluación pre y post ensayo para relevar el conocimiento de los participantes. La evaluación fue desarrollada en base a las guías de recomendaciones clínicas vigentes sobre el manejo de la hipertensión arterial.

El grupo intervención recibía correos electrónicos interactivos con contenido educativo en forma de preguntas de opción múltiple contextualizadas mediante escenarios clínicos, mientras que el grupo control recibía sólo el contenido en forma de texto. Las preguntas de opción múltiple utilizaban aprendizaje por repetición espaciada (en inglés spaced education) y mecánicas de juego como progresión dinámica y comparaciones del desempeño del participante en relación a los otros participantes. El grupo intervención recibió una pregunta cada tres días, la mecánica de progresión dinámica constaba en reenviar preguntas a los 12 o 24 días dependiendo de si respondían correctamente o no, respectivamente.

\section{Medición de resultados principales}

El tiempo en llegar a la TA deseada $(<140 / 90 \mathrm{~mm} \mathrm{Hg})$ fue el resultado principal. Los valores de TA de los pacientes se obtuvieron de la práctica médica cotidiana.

\section{Resultados Principales}

El $87 \%$ del grupo intervención (48/55) contestó todas las preguntas y un $84 \%$ del grupo control (47/56) leyó los textos. En un análisis multivariable de 14.336 pacientes, se encontró que el hazard ratio del tiempo en llegar en menor tiempo a la TA deseada en el grupo intervención fue de 1,043 (IC95\% 1,007 a $1,081 ; P=0,018)$. Por cada médico que participó de la intervención, 2,3 pacientes más alcanzaron la meta de TA.

\section{Conclusiones}

Un juego online con aprendizaje por repetición espaciada generó una pequeña pero significativa reducción en el tiempo que tardan los médicos de atención primaria en llevar la TA de sus pacientes al valor deseado.

Fuente de financiamiento: Veterans Affairs Health Services Research and Development Service.

\section{Comentario}

El término Gamification describe el uso de elementos de juego en contextos no lúdicos para mejorar la experiencia del usuario'. Si bien en los últimos años se incorporaron juegos y elementos de juegos al área de salud2 ${ }^{2,4}$, aún hay poca evidencia de su utilidad y efectividad. En este estudio se incorporaron elementos de juego con el fin de evaluar su eficacia en educar e incentivar la acción por parte del médico. Si bien los resultados son estadísticamente significativos esto no es necesariamente indicativo de una intervención efectiva. La diferencia en la frecuencia de notificaciones actuando como recordatorios entre el grupo intervención y el grupo control puede haber actuado como confundidor. Es posible que los médicos del grupo intervención hayan actuado distinto sólo por tener más presente la necesidad de llevar a sus pacientes a la tensión arterial ideal. Un tercer grupo que contemple esta altemativa hubiera sido apropiado para evaluar esta posibilidad.

\section{Conclusiones del comentador}

Hay quienes consideran que la creciente popularidad de los elementos de juego en salud es sólo una moda pasajera ${ }^{5}$, sin embargo se siguen reportando datos de intervenciones eficaces ${ }^{6,7.8}$. Consideramos que el uso de la gamification como método para facilitar el aprendizaje e incentivar la acción amerita más estudios de investigación que exploren su potencial.

Guido Giunti y Analía Baum [ Departamento de Informática del Hospital Italiano de Buenos Aires. guido.giunti@hospitalitaliano.org.ar ]

Baum A, Giunti G. Un juego educativo online para médicos ayuda a que sus pacientes alcancen más rápidamente las metas de control de la presión arterial. Evid Act Pract Ambul. 2015;18(3):74. Jul-Sep. Comentado de: Kerfoot BP, y col. An online spaced-education game among clinicians improves their patients' time to blood pressure control: a randomized controlled trial. Circulation. 2014;7(3):468-74. PMID: 24847084 ..

\section{Referencias}

1. Kapp KM. The Gamification of Learning and Instruction: Game- Based Methods and Strategies for Training and Education. San Francisco, CA: Pfeiffer; 2012.

2. Boulos M N K, \& Yang SP. Exergames for health and fitness: the roles of GPS and geosocial apps. International Journal of Health Geographics 2013;12(1):18.

3. Primack BA, y col. Role of video games in improving health-related outcomes: a systematic review. Am J Prev Med 2012;42:630-8.

4. Cafazzo JA, y col. Design of an mHealth app for the self-management of adolescent type 1 diabetes: a pilot study. J Med Internet Res. 2012;14(3):e70.

5. Lister C, y col. Just a Fad? Gamification in Health and Fitness Apps. JMIR Serious Games 2014;2(2):e9.

6. Jones BA, y col. The FIT Game: preliminary evaluation of a gamification approach to increasing fruit and vegetable consumption in school. Prev Med. 2014 Nov;68:76-9.

7. Kanthan R, Senger JL. The impact of specially designed digital games-based learning in undergraduate pathology and medical education. Arch Pathol Lab Med. 2011;135(1):135-42.

8. Lohse K. y col. Video games and rehabilitation: using design principles to enhance engagement in physical therapy. Journal of Neurologic Physical Therapy 2013;37(4):166-75. 